\title{
BMJ Open Non-pharmacological interventions for treating sexual dysfunction in postpartum women: a systematic review protocol
}

\author{
Michelly Nóbrega Monteiro, ${ }^{1}$ Kleyton Santos Medeiros, ${ }^{1}$ laponira Vidal, ${ }^{1}$ \\ Ivete Matias, ${ }^{1}$ Ricardo Ney Cobucci, ${ }^{1,2}$ Ana Katherine Gonçalves ${ }^{1}$
}

To cite: Monteiro MN, Medeiros KS, Vidal I, et al. Non-pharmacological interventions for treating sexual dysfunction in postpartum women: a systematic review protocol. BMJ Open 2019;9:e028660. doi:10.1136/ bmjopen-2018-028660

- Prepublication history for this paper is available online. To view these files, please visit the journal online (http://dx.doi org/10.1136/bmjopen-2018028660).

Received 06 March 2019 Revised 31 July 2019

Accepted 05 September 2019

Check for updates

(c) Author(s) (or their employer(s)) 2019. Re-use permitted under CC BY-NC. No commercial re-use. See rights and permissions. Published by BMJ.

${ }^{1}$ Health Sciences Postgraduation Program, Universidade Federal do Rio Grande do Norte, Natal, Brazil

${ }^{2}$ Biotechnology Postgraduation Program, Universidade Potiguar, Natal, Brazil

\section{Correspondence to}

Dr Ana Katherine Gonçalves; anakatherine_ufrnet@yahoo. com.br

\section{ABSTRACT}

Introduction Sexual dysfunction in the postpartum period is a very common and relevant clinical problem, which has a significant adverse impact on the health of women. We aim to analyse the efficacy and safety of non-pharmacological interventions for treating sexual dysfunction in postpartum women. Our review aims to provide accurate data for effective policy-making and improve our understanding of the treatment of postpartum sexual dysfunction with non-pharmacological therapies. Methods and analysis The Cochrane Central Register of Controlled Trials in The Cochrane Library, clinicaltrials. gov, Medline/PubMed, CINAHL (Cumulative Index to Nursing and Allied Health Literature), LILACS (Literatura Latino-americana e do Caribe em Ciências da Saúde) and Embase will be used to search for articles dated from database inception to July 2019. Randomised controlled clinical trials and large prospective cohorts with control groups using non-pharmacological treatments for sexual dysfunction in postpartum women will be included. Sexual problems are directly linked to sexual dysfunction; thus, the primary outcome will be the absolute number or percentage of sexual issues in each treatment group. The secondary outcomes will be assessed by decreased sexual problems, such as lack of lubrication, decreased libido and difficulty reaching orgasm. Three reviewers will independently select trials and extract data from the original publications. The citations will be screened independently by reviewers in duplicate. The risk of bias of the included studies will be assessed according to the Cochrane risk of bias tool. Data synthesis will be performed using Review Manager (RevMan) software V.5.2.3. In the event that a meta-analysis is possible, we will assess the heterogeneity across the studies by computing the $\mathrm{I}^{2}$ statistic.

Ethics and dissemination As the design of this study includes a review of published data, the need to obtain ethical approval was waived by our institutional review committee. We intend to publish the findings of this systematic review in a peer-reviewed journal. PROSPERO registration number CRD42018103077.

\section{Strengths and limitations of this study}

- There are no existing reviews regarding the use of non-pharmacological interventions for treating sexual dysfunction in postpartum women.

- This systematic review includes studies on postpartum female participants of all ages who have sexual dysfunction problems.

- Three reviewers will independently select trials that are eligible for inclusion in this review, extract data on different variables and assess the risk of bias.

- The review will be limited by variation in sexual disorders, such as the presence of pain, difficulty reaching orgasm, lack of arousal, poor lubrication and low desire.

- Our review and meta-analysis intend to combine and compute the results of different studies that have comparable effect sizes; however, we acknowledge the risk of only obtaining a limited number of studies with small sample sizes.

\section{INTRODUCTION}

\section{Description of the condition}

Female sexual dysfunction is highly prevalent and takes many different forms including lack of sexual desire, impaired arousal, inability to achieve orgasm, pain with sexual activity or a combination of these issues. ${ }^{12}$ The reasons for female sexual dysfunction are multifactorial, often with several different aetiologies contributing to the problem. The postpartum period is characterised by not only physical and emotional changes but also a specific shift in social interactions; this includes the couple's relationship, which can affect their sex life in an abrupt manner. ${ }^{1-3}$ During this transitional phase, balance and flexibility are required of the couple, and it can also be an exciting time to develop a new perspective on sexuality. A couple's sexual life after childbirth might either improve or experience changes that negatively impact on the couple's physical and psychological health. ${ }^{2-5}$ 
There may be decreased vaginal lubrication caused by the high concentration of progesterone, which often results in discomfort/pain during vaginal penetration. ${ }^{6-8}$ With regards to sexuality, the couple may have decreased desire and frequency of sexual activity, but in some cases the desire is increased. Furthermore, there may be changes in the choice of sexual positions, in particular when discomfort/pain is felt; the latter can reduce eroticism and sexual performance ${ }^{7-9}$ The most common female sexual problem after childbirth is dyspareunia, or pain during vaginal penetration, especially after the first pregnancy and in cases where the female has undergone an episiotomy. ${ }^{9-13}$

\section{Description of the intervention}

Sexual disorders can be complex, and their treatment can be time-intensive and require special expertise. Non-pharmacological therapies, such as sexual and couples' therapy, pelvic floor exercises, psychotherapy, lifestyle changes, improving body image, and use of vaginal lubricants and moisturisers are extremely important in the postpartum period.

Ideally, with the women's prior consent, communication and management decisions should be shared between the patient's clinician and other healthcare providers who treat the patient (eg, the physiotherapist).

\section{How the intervention might work}

Female sexual dysfunction typically affects more than one aspect of sexuality (eg, orgasm, desire and arousal), and most therapies also impact several aspects. Thus, it is not generally possible to identify an isolated sexual issue and select a therapy that specifically targets that concern. ${ }^{11-21}$

Many studies of sexual dysfunction treatment use validated questionnaire scores $^{22-25}$ as a measurement of outcome. Since there are multiple questionnaires that use different questions and scales, it is difficult to compare data between studies and treatments.

The purpose of this study is to compare non-pharmacological interventions and/or therapies available during the postpartum period and determine the efficacy of each of them either separately or combined, with the objective to decrease sexual problems in women after childbirth.

\section{Why it is important to perform this review}

Postpartum sexual dysfunction is not a rare condition; indeed, a longitudinal study found that $26.5 \%-34.8 \%$ of women were at risk of sexual dysfunction in pregnancy and puerperium. ${ }^{26}$ Factors beyond delivery that can contribute to postpartum sexual dysfunction include perineal trauma (surgical or non-surgical) ${ }^{14-16}$; emergency caesarean delivery or vacuum-assisted vaginal delivery ${ }^{11}{ }^{12}$; low oestrogen and lubrication levels, particularly in breastfeeding women ${ }^{14-18}$ and postpartum mood changes, fatigue and time constraints. ${ }^{19}$ Although studies have reported that certain sexual problems reported by postpartum women, such as lack of vaginal lubrication and pain during intercourse, returned to levels similar to those found before pregnancy at approximately 12 months postpartum, the rate of decreased interest in sexual activity remained higher in these patients. ${ }^{20-2527}$

The WHO considers sexual dysfunction a major public health problem that, given the negative impact that it has been shown to have on quality of life, should be thoroughly investigated. ${ }^{20-13}$

\section{OBJECTIVES}

The objective of the study is to systematically review the scientific literature and, if possible, perform a meta-analysis to determine the effectiveness of different non-pharmacological interventions for treating sexual dysfunction in postpartum women.

\section{METHODS}

The Preferred Reporting Items for Systematic Reviews and Meta-Analyses (PRISMA) statement guidelines will be used to construct this systematic review protocol. ${ }^{28}$

\section{Criteria for considering studies for this review \\ Types of studies}

The inclusion criteria will be as follows: (1) articles will be considered without any language restrictions; (2) studies including postpartum women, irrespective of age; (3) if the data subsets are published in more than one article, only the latest subset will be included and (4) parallel randomised controlled trials (RCTs) and large prospective cohorts with control groups. The following studies will be excluded: (1) case reports; (2) publications that do not specifically relate to sexual dysfunction in women and (3) those with insufficient data to be extracted or calculated from the original article.

\section{Types of participants}

Female participants with sexual issues initiated in pregnancy or in the puerperium, irrespective of age, will be included. Analyses of trials based on sexual dysfunction in other conditions, such as menopause, will be excluded.

\section{Types of interventions}

Parallel RCTs and cohort studies that compare non-pharmacological rehabilitative interventions (such as couples' therapy, sex therapy and pelvic floor exercises) with a concurrent control group receiving no treatment or a placebo will be eligible. Studies comparing these therapies with another active intervention will be excluded.

\section{Types of outcome measurements}

The Female Sexual Function Index ${ }^{21} 22$ is a multidimensional self-reporting instrument for the assessment of female sexual function that has been developed as a quick tool for assessing the key dimensions of sexual function in women (desire, arousal, lubrication, orgasm, satisfaction and pain). The primary outcome will be sexual problem improvement rates (directly linked to sexual dysfunction) in each treatment group. The secondary outcome will be 
the length of time of sexual dysfunction, defined as the presence of pain, more difficulty reaching orgasm, lack of arousal, poor lubrication and low desire; the outcome measurements will be based on percentages. Small but significant changes will be considered clinically relevant because a sexual upgrade almost always equates to an improvement in the quality of life. Any discrepancies in outcome measurements will be resolved through discussion by the review team.

\section{Patient and public involvement}

This work is a systematic review protocol; the research will be performed by a wide and comprehensive search of literature from databases, and individual patient data will not be included. Thus, the authors will not involve patients when setting the search questions, determining the outcome measurements, during the design and implementation of the study and in the dissemination of the results.

\section{Search methods for identification of studies \\ Electronic searches}

The Cochrane Central Register of Controlled Trials in The Cochrane Library, clinicaltrials.gov, Medline/ PubMed, CINAHL, LILACS and Embase will be used to search for articles dated from database inception to July 2019.

\section{Other sources}

The scope of the computerised literature search may be enlarged based on the reference lists of retrieved articles.

\section{Search strategy}

Box 1 presents the search strategy for Medline.

\section{Data collection and analysis}

\section{Selection of studies}

Three authors, MNM, KSM and AKG will independently screen the search results using titles and abstracts. Duplicates and reviews will be removed from the database. Reviewers will then go through the full text in order to determine whether it meets the inclusion criteria. Studies will be excluded if women were not in the postpartum period. Discrepancies will be resolved by a fourth reviewer, IV. The selection of the study is summarised in a PRISMA flow diagram (figure 1).

\section{Data extraction and management}

Three review authors, MNM, KSM and AKG, will independently assess and extract the included study data according to a data extraction form that includes basic details (name of the authors, publication date, country and sample size), participant details (age and underlying symptomatology), diagnostic standards (lack of orgasm, desire and arousal disorders, vaginal discomfort/pain and poor lubrication), intervention details (sex and couples therapy, pelvic floor exercises, psychotherapy, lifestyle changes, improving body image, use of lubricants and moisturisers) and outcomes (decreased rates of sexual

\section{Box 1 Medline search strategy}
Search items
1. Randomized controlled trial
2. Controlled clinical trial
3. Cohort studies
4. Clinical trial
5. $0 R / 1-4$
6. Sexual dysfunction
7. Female sexual dysfunction
8. Hypoactive sexual desire disorder
9. Psychosexual dysfunctions
10. Sexual arousal disorder
11. $\mathrm{OR} / 6-10$
12. Postpartum sexual function
13. Postpartum period
14. Pregnancy postpartum
15. Postnatal care
16. Postpartum pain
17. Postpartum pelvic floor
18. Puerperium
19. $\mathrm{OR} / 12-18$
20. Non-pharmacological therapies
21. Treatment outcome
22. Couples therapy
23. Sexual therapy
24. Sexual health
25. Sexual satisfaction
26. $\mathrm{OR} / 20-25$
27. Pelvic floor
28. Psychotherapy
29. Exercise movement techniques
30. Body image
31. Vaginal lubricants
32. Vaginal moisturizers
33. $\mathrm{OR} / 27-32$
34. 5 AND 11 AND 19 AND 26 AND 33

dysfunction or recurrence rates of sexual dysfunction). Extracted data will be checked by RNC and disagreements will be resolved through discussion. If necessary, a further reviewer, IV, will provide the final judgement.

\section{Risk of bias assessment}

Two independent reviewers, MNM and RNC, will independently assess the risk of bias in the included studies using the Cochrane risk of bias tool. ${ }^{29}$ The modified Cochrane Collaboration tool will be used to assess the risk of bias for RCTs. Bias is assessed as a judgement (high, low or unclear) for individual element from five domains (selection, performance, attrition, reporting and other).

\section{Measures of treatment effect}

The primary outcome (improvement rate in sexual function) will be based on percentages. In addition, the dichotomous outcomes (improvement or no improvement) will be included in this research. Any small but significant change will be considered to be clinically relevant, since this study is about the quality of sexual life. This will be carried out using Review Manager (RevMan) software 


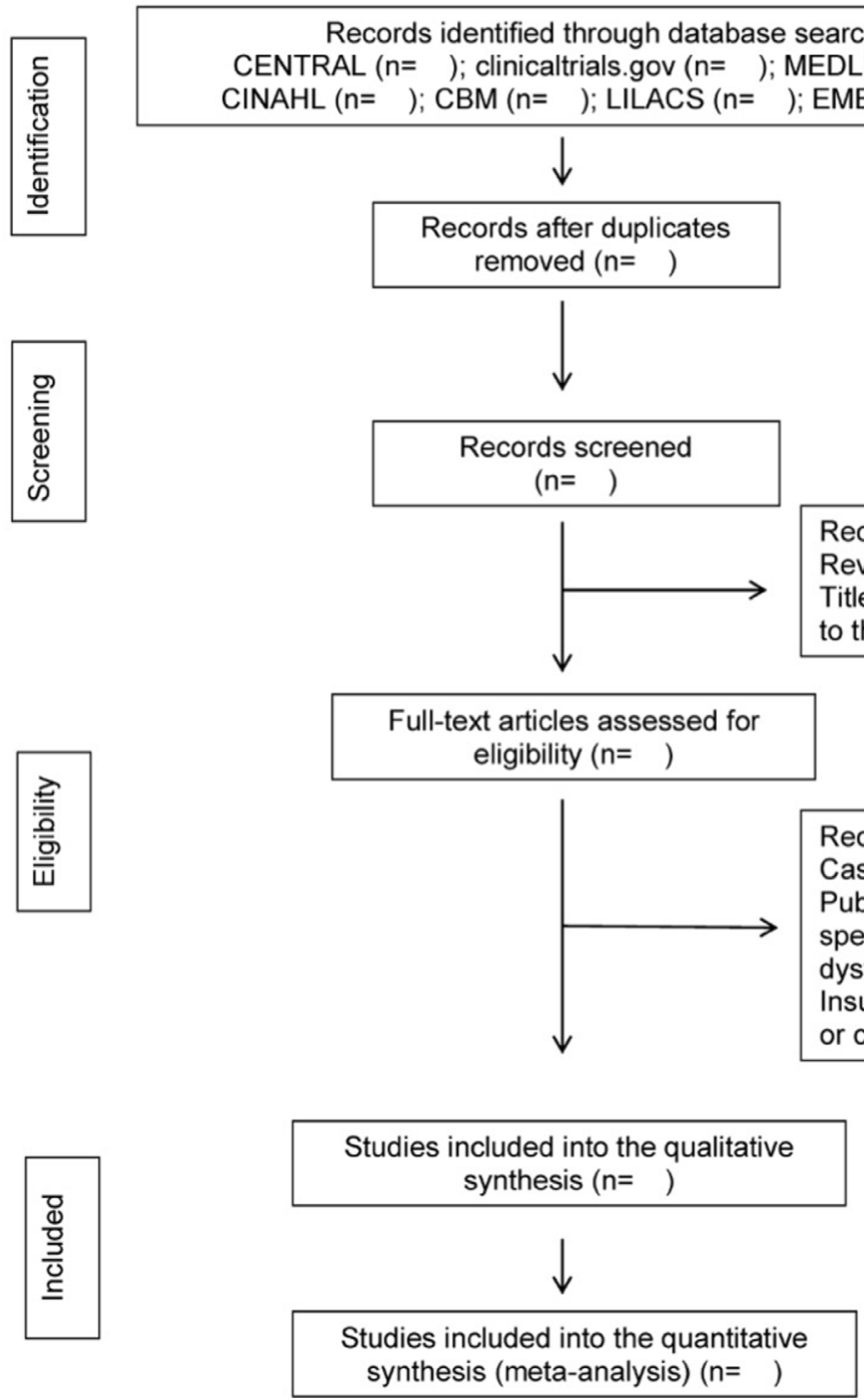

Figure 1 Flow diagram of the search for eligible studies on non-pharmacological interventions for treating sexual dysfunction in postpartum women. CENTRAL: Cochrane Central Register of Controlled Trials.

V.5.2.3. We will calculate the OR for dichotomous data and the weight mean difference, with associated $95 \% \mathrm{CI}$, for continuous data.

\section{Unit of analysis issues}

For a decreased rate of sexual dysfunction, the unit of analysis will be defined as 21 and 30 days after the initiation of therapy. For the length of time of sexual dysfunction, 3 months and 6 months following the intervention will be considered as short-term and long-term follow-up, respectively.

\section{Addressing missing data}

We will attempt to obtain any missing data by contacting the first or corresponding authors or coauthors of an article via phone, email or post. If we fail to receive any necessary information, the data will be excluded from our analysis and will be addressed in the discussion section. An alternative way to achieve a balanced dataset instead of discarding units that have incomplete observations is to estimate them using imputation-based rules; this method can predict missing values to complete the data and then analyse the obtained data through standard statistical methods. Through the simple imputation method each missing value is replaced by a single imputed value. The completed data sets are analysed and used to estimate a plausible value representing the uncertainty about the value to be imputed. The included papers will also have their references screened for additional studies that were not identified in the electronic searches.

\section{Assessment of heterogeneity}

The heterogeneity between the trial results will be evaluated using a standard $\chi^{2}$ test with a significance level of $\mathrm{p}<0.1$. To assess heterogeneity, we plan to compute the $\mathrm{I}^{2}$ statistic, which is a quantitative measurement of inconsistency across studies. A value of $0 \%$ indicates no observed heterogeneity, whereas $\mathrm{I}^{2}$ values $\geq 50 \%$ indicate a substantial level of heterogeneity. However, the assessment of heterogeneity will only occur if it is necessary in order to undertake a meta-analysis. 


\section{Assessment of reporting biases}

Considering that this protocol has not yet been initiated, the number of studies that will be found is still undetermined. However, if 10 or more publications that pertain to the measured outcome are found, funnel plots, as well as the tests of Egger and Begg, will be used to assess the presence of heterogeneity and potential reporting biases. ${ }^{30} \mathrm{~A}$ linear regression approach will be used to evaluate funnel plot asymmetry.

\section{Data synthesis}

This will be carried out using the RevMan software V.5.2.3; this allows the user to enter protocols, complete reviews, as well as include text, characteristics of the studies, comparison tables and study data. The RevMan software will allow us to perform meta-analyses of the data that the ORs will obtain. For dichotomous outcomes, we will extract or calculate the OR and $95 \%$ CI for each study. Where there is heterogeneity $\left(\mathrm{I}^{2}>75 \%\right)$, a random-effect model will be used to combine the trials to calculate the relative risk (RR) and 95\% CI, using the DerSimonian-Laird algorithm in The Meta for Package, a meta-analysis package for $\mathrm{R}$.

Other study characteristics and results will be summarised narratively if the meta-analysis cannot be performed for all or some of the included studies.

\section{Sensitivity analyses}

We will conduct sensitivity analyses for the primary outcome in order to determine whether the outcome would be different if the analyses of subgroups considering different non-pharmacological therapies were performed, as well as if we included only clinical trials involving postpartum women at an age where the prevalence of sexual dysfunction is higher. Sensitivity analyses will be important to explore the robustness of the findings regarding the study quality and sample size, and this is only possible to consider if a meta-analysis is undertaken. This will be shown in a summary table.

\section{Subgroup analyses}

Subgroup analyses will be based on the type of intervention, type of delivery, participant ages, number of deliveries and study settings. Meta-regressions will be conducted to compare the ratio of RRs to investigate whether any observed differences between the subgroups are statistically significant.

\section{Confidence in cumulative evidence}

To describe the strength of evidence for the included data, we will use the Grading of Recommendation Assessment, Development and Evaluation (GRADE) approach as outlined in the GRADE handbook ${ }^{31}$ to incorporate summary assessments into broader measurements to ensure the judgments about bias risk, consistency, directness, precision and publication bias. ${ }^{27}$ The quality of evidence will be identified as high (the true effect lies close to that of the estimate of the effect), moderate (the true effect is likely to be close to the estimate of the effect, but there is a possibility that it is substantially different), low (the true effect may be substantially different from the estimate of the effect) or very low (the true effect is likely to be substantially different from the estimate of the effect).

\section{DISCUSSION}

Sexual dysfunction in the postpartum period is a very common and relevant clinical problem, with a significant adverse impact on women's health. ${ }^{2911-15}$ We aim to analyse the efficacy and safety of interventions for treating sexual dysfunction in postpartum women. We expect that our review will provide accurate data to assist with effective policy-making. Furthermore, this review will improve our understanding of the treatment for postpartum sexual dysfunction with non-pharmacological therapies. Research on female sexuality during the postpartum period is of extreme importance; however, many professionals neglect the emotional and sexual needs of these patients. ${ }^{812-18}$

\section{Ethics and dissemination}

The need for ethical approval was waived because this systematic review will use published patient data. Findings of this systematic review will be published in a peer-reviewed journal, and updates will be conducted if there is a sufficient amount of new evidence that would likely alter the conclusions of the review.

Acknowledgements The authors acknowledge the assistance provided by the Graduate Program for Health Sciences of the Federal University of Rio Grande do Norte (UFRN) in the guidance of literary research.

Contributors MNM is the guarantor. IV and IM contributed to the conception of this review. MNM and KSM drafted the manuscript of the protocol, and AKG revised it. RNC and AKG developed the search strategies, and AKG and KSM will implement them. MNM, KSM, IV, IM, RNC and AKG will screen the potential studies, extract the data and assess their quality. In case of disagreement between the data extractors, RNC will advise on the methodology and will work as the arbitrator. MNM will complete the data synthesis. All authors have approved the final version for publication.

Funding The authors have not declared a specific grant for this research from any funding agency in the public, commercial or not-for-profit sectors.

Competing interests None declared.

Patient consent for publication Not required.

Provenance and peer review Not commissioned; externally peer reviewed.

Data availability statement Data are available in a public, open access repository. There are no data in this work. Data are available upon reasonable request. Data may be obtained from a third party and are not publicly available. No data are available. All data relevant to the study are included in the article or uploaded as supplementary information.

Open access This is an open access article distributed in accordance with the Creative Commons Attribution Non Commercial (CC BY-NC 4.0) license, which permits others to distribute, remix, adapt, build upon this work non-commercially, and license their derivative works on different terms, provided the original work is properly cited, appropriate credit is given, any changes made indicated, and the use is non-commercial. See: http://creativecommons.org/licenses/by-nc/4.0/.

\section{REFERENCES}

1 Yıldız $\mathrm{H}$. The relation between prepregnancy sexuality and sexual function during pregnancy and the postpartum period: a prospective study. J Sex Marital Ther 2015;41:49-59. 
2 Shifren JL, Monz BU, Russo PA, et al. Sexual problems and distress in United States women: prevalence and correlates. Obstet Gynecol 2008;112:970.

3 Monteiro MN, Lucena EEdeS, Cabral PU, et al. Prevalence of sexual dysfunction among expectant women. Rev Bras Ginecol Obstet 2016;38:559-63.

4 Vettorazzi J, Marques F, Hentschel $\mathrm{H}$, et al. [Sexuality and the postpartum period: a literature review] Portuguese. Rev HCPA 2012;32:473-9.

5 Ribeiro MC, Nakamura MU, Torloni MR, et al. Maternal overweight and sexual function in pregnancy. Acta Obstet Gynecol Scand 2016;95:45-51.

6 Prado DS, Lima RV, de Lima LMMR. [Impact of pregnancy on female sexual function]. Rev Bras Ginecol Obstet 2013;35:205-9.

7 Johnson CE. Sexual health during pregnancy and the postpartum. $J$ Sex Med 2011;8:1267-84. quiz 1285-6..

8 Leeman LM, Rogers RG. Sex after childbirth: postpartum sexual function. Obstet Gynecol 2012;119:647-55.

9 Yee LM, Kaimal AJ, Nakagawa S, et al. Predictors of postpartum sexual activity and function in a diverse population of women. $J$ Midwifery Womens Health 2013;58:654-61.

10 Lowenstein L, Mustafa S, Burke Y. Pregnancy and normal sexual function. are they compatible? J Sex Med 2013;10:621-2.

11 Barbara G, Pifarotti P, Facchin F, et al. Impact of mode of delivery on female postpartum sexual functioning: spontaneous vaginal delivery and operative vaginal delivery vs. cesarean section. J Sex Med 2016;13:393-401.

12 Laganà AS, Burgio MA, Ciancimino L, et al. Evaluation of recovery and quality of sexual activity in women during postpartum in relation to the different mode of delivery: a retrospective analysis. Minerva Ginecol 2015;67:315-20.

13 Faisal-Cury A, Menezes PR, Quayle J, et al. The relationship between mode of delivery and sexual health outcomes after childbirth. J Sex Med 2015;12:1212-20.

14 McDonald EA, Gartland D, Small R, et al. Dyspareunia and childbirth: a prospective cohort study. BJOG: Int J Obstet Gy 2015;122:672-9.

15 Wesnes SL, Lose G. Preventing urinary incontinence during pregnancy and postpartum: a review. Int Urogynecol J 2013;24:889-99.

16 Martin-Martin S, Pascual-Fernandez A, Alvarez-Colomo C, et al. Urinary incontinence during pregnancy and postpartum. associated risk factors and influence of pelvic floor exercises. Arch Esp Urol 2014;67:323-30.
17 Pelaez M, Gonzalez-Cerron S, Montejo R, et al. Pelvic floor muscle training included in a pregnancy exercise program is effective in primary prevention of urinary incontinence: a randomized controlled trial. Neurourol Urodyn 2014;33:67-71.

18 Hall B, Woodward S. Pelvic floor muscle training for urinary incontinence postpartum. Br J Nurs 2015;24:576-9.

19 Sahakian J, Woodward S. Stress incontinence and pelvic floor exercises in pregnancy. Br J Nurs 2012;21:S10-5.

20 Findik RB, Unluer AN, Sahin E, et al. Urinary incontinence in women and its relation with pregnancy, mode of delivery, connective tissue disease and other factors. Adv Clin Exp Med 2012;21:207-13.

21 Wiegel M, Meston C, Rosen R. The female sexual function index (FSFI): cross-validation and development of clinical cutoff scores. $J$ Sex Marital Ther 2005;31:1-20.

22 Pacagnella RdeC, Vieira EM, Rodrigues OM, et al. [Cross-cultural adaptation of the Female Sexual Function Index]. Cad Saude Publica 2008;24:416-26.

23 Hentschel H, Alberton DL, Capp E, et al. [Validation of the Female Sexual Function Index (FSFI) for use in Portuguese]. Rev HCPA 2007;27:10-14

24 Thiel RdoRC, Dambros M, Palma PCR, et al. [Translation into Portuguese, cross-national adaptation and validation of the Female Sexual Function Index]. Rev Bras Ginecol Obstet 2008;30:504-10.

25 Sarwer DB, Durlak JA. A field trial of the effectiveness of behavioral treatment for sexual dysfunctions. J Sex Marital Ther 1997;23:87-97.

26 Wallwiener S, Müller M, Doster A, et al. Sexual activity and sexual dysfunction of women in the perinatal period: a longitudinal study. Arch Gynecol Obstet 2017;295:873-83.

27 Billups KL, Berman L, Berman J, et al. A new non-pharmacological vacuum therapy for female sexual dysfunction. J Sex Marital Ther 2001;27:435-41.

28 Moher D, Liberati A, Tetzlaff J, et al. Preferred reporting items for systematic reviews and meta-analyses: the PRISMA statement. BMJ 2009;339:b2535.

29 Higgins JPT, Altman DG, Gøtzsche PC, et al. The Cochrane collaboration's tool for assessing risk of bias in randomised trials. BMJ 2011;343:d5928.

30 Egger M, Davey Smith G, Schneider M, et al. Bias in meta-analysis detected by a simple, graphical test. BMJ 1997;315:629-34.

31 Balshem H, Helfand M, Schünemann HJ, et al. Grade guidelines: 3. rating the quality of evidence. J Clin Epidemiol 2011;64:401-6. 